\title{
DERMATOMIOSITE COMO DIAGNÓSTICO DIFERENCIAL DE DEPRESSÃO MAIOR: RELATO DE CASO
}

\section{NATÁLIA TOMICH DE PAIVA MIRANDA ${ }^{1}$, LUCIANE DE ANDRADE ROCHA ${ }^{2}$, MAIÍRA DE PAIVA BARBOSA ${ }^{3}$, VINÍCIUS SCHAMMAS PENATTI ${ }^{4}$.}

\author{
1 Doutora em Bioquímica pela Universidade Federal de Minas Gerais. Professora no Centro Univeristário \\ UNIFACIG.ntomich@gmail.com. \\ 2 Graduanda de Medicina pelo Centro Universitário UNIFACIG. lucianearocha@gmail.com. \\ 3 Graduanda de Medicina pelo Centro Universitário UNIFACIG. maira_pb@ hotmail.com. \\ 4 Graduando de Medicina pelo Centro Universitário UNIFACIG. viniciuspenatti@gmail.com.
}

\section{RESUMO}

A dermatomiosite (DM) é uma doença que acomete o tecido conjuntivo cuja etiologia é de origem desconhecida, podendo ser subaguda, aguda e crônica. A doença tem início insidioso com evolução lenta, apresentando edema de membros superiores e inferiores seguido de dermatite e acometimento muscular. Os principais sintomas são fraqueza muscular proximal, principalmente do tríceps e quadríceps, ocorrendo de forma simétrica, além de exantemas cutâneas característicos. As principais complicações da DM são perda dos reflexos tendinosos profundos, atrofia muscular, disfonia, disfagia e elevado risco para doença pulmonar intersticial. A principal forma de diagnóstico é através do exame clínico, devendo observar as pápulas de Gottron, as quais são consideradas sinais patognomônicos, também deve-se aplicar critérios diagnósticos específicos, podendo ser complementados por exames laboratoriais e de imagem, os quais se destacam a dosagem de creatinocinase e a eletromiografia. O tratamento é realizado com glicocorticoide, possibilitando bom prognóstico e controle da doença. $\mathrm{O}$ caso relatado e análise de publicações levantadas trazem à luz a discussão da importância de se fazer um diagnóstico precoce para definição de uma adequada terapêutica para dermatomiosite, com o intuito de evitar a evolução para complicações, as quais podem se tornar irreversíveis, bem como utilizar o caso clínico para corroborar com as informações disponíveis pela literatura, tanto em termos de diagnóstico como de terapêutica. As informações foram levantadas com base na entrevista com a paciente e sua acompanhante, revisão do prontuário, revisão sistemática de artigos e literaturas específicas, as quais envolveram livros, cartilhas e protocolos específicos para a patologia abordada.

Palavras-chave: Dermatomiosite; Epidemiologia; Miopatias Inflamatórias.

\section{DERMATOMIOSITE AS DIFERENTIAL DIAGNOSIS OF LARGER DEPRESSION: CASE REPORT}

\section{ABSTRACT}


Dermatomyositis (DM) is a disease that affects the connective tissue whose etiology is of unknown origin and may be subacute, acute and chronic. The disease has an insidious onset with slow evolution, presenting edema of the upper and lower limbs followed by dermatitis and muscular involvement. The main symptoms are proximal muscle weakness, mainly of the triceps and quadriceps, occurring symmetrically, as well as characteristic skin rashes. The main complications of DM are loss of deep tendon reflexes, muscle atrophy, dysphonia, dysphagia and high risk for interstitial lung disease. The main form of diagnosis is through clinical examination, and Gottron's papules, which are considered pathognomonic signs, should be observed. Specific diagnostic criteria should also be applied, and may be complemented by laboratory and imaging tests, such as dosage. creatinokinase and electromyography. The treatment is performed with glucocorticoid, allowing good prognosis and disease control. The case report and the analysis of publications raised bring to light the discussion of the importance of making an early diagnosis to define an appropriate therapy for dermatomyositis, in order to avoid the evolution to complications, which may become irreversible, as well as to use the clinical case to corroborate the information available in the literature, both in terms of diagnosis and therapy. The information was collected based on the interview with the patient and her companion, medical record review, systematic review of articles and specific literature, which involved books, booklets and specific protocols for the pathology addressed.

Keywords: Dermatomyositis; Epidemiology; Inflammatory myopathies

\section{INTRODUÇÃO}

A dermatomiosite (DM) é uma doença que acomete o tecido conjuntivo cuja etiologia é de origem desconhecida, podendo ser subaguda, aguda e crônica. A doença tem início insidioso com evolução lenta, apresentando edema de membros superiores e inferiores seguido de dermatite e acometimento muscular (GIÁCOMO et al., 2010).

A DM pode ter relação com antígenos de histocompatibilidade (HLA), sendo classificada em dois grandes grupos imunogenéticos: os de melhor prognóstico (HLADRw53) e os de pior prognóstico (HLA-DRw52). O aspecto hereditário, apesar de raro, foi relatado em alguns casos. Além disso, a doença pode ser precipitada por infecções virais, tais como hepatite B, influenza A e HIV, bem como o uso de algumas substâncias, tais como penicilina, antiinflamatórios não-esteroidais, corticoides, álcool e estatinas (ORTIGOSA et al., 2008).

A principal manifestação clínica é a fraqueza muscular proximal, principalmente do tríceps e quadríceps, ocorrendo de forma simétrica, além de exantemas cutâneas característicos. As manifestações cutâneas patognomônicas, como as pápulas de Gottron e o sinal de Gottron, podem estar presentes em $70 \%$ dos casos, bem como outros achados, heliotropo, edema periorbital, eritema maculoso e linhas escurecidas fissuradas 
nas regiões lateral e palmar dos dedos, denominadas "mãos de mecânico" (ORTIGOSA et al., 2008).

A DM possui incidência média anual de dois a sete novos casos por milhão de habitantes. É uma doença bimodal com pico entre 10 e 15 anos e de 45 a 60 anos, sendo a idade média de diagnóstico, 40 anos, sendo, em geral, as mulheres duas vezes mais acometidas que os homens (PEREIRA et al., 2011).

Existe uma relação entre dermatomiosite e neoplasias em adultos, principalmente nos idosos, tornando o prognóstico desfavorável, uma vez que aumenta a morbimortalidade da doença (GIÁCOMO et al., 2010). A dermatomiosite neoplásica tem início e evolução mais agudos, com manifestações cutâneas precoces e intensas, além da presença de necrose. Nas mulheres, o câncer mais comumente encontrado é o de ovário, já nos homens, destacam-se o de testículo, próstata, estômago, o linfoma nãoHodgkin e o esofágico (VILAS et al., 2009).

$\mathrm{O}$ presente relato tem por objetivo descrever o caso de uma paciente com dermatomiosite. As informações foram obtidas pela anamnese com a própria paciente e sua acompanhante.

\section{METODOLOGIA}

O presente estudo trata-se de um relato de caso em que se discutem as características da dermatomiosite, incluindo sua fisiopatologia, manifestações clínicas, abordagens diagnósticas e tratamento.

Para realização deste estudo, foram consultadas plataformas: SciELO (ScientificElectronic Library Online) e PubMed (National Library of Medicine e do NationalInstitutesof Health) explorando artigos publicados entre 2008 e 2019, buscando, dessa forma, identificar o que os atuais estudos dizem sobre a temática. Textos acadêmicos e livros de autores considerados referência na área também foram utilizados. O método foi baseado na análise das publicações pertinentes ao assunto.

Inicialmente, o total de 16 artigos foram selecionados através do título, resumo e palavras-chaves para determinar a sua possível inclusão no estudo. A partir daí, 7 artigos, que cumpriam os objetivos da pesquisa, foram selecionados. Como estratégia de pesquisa foram empregados os mesmos descritores em todos os bancos de dados. 
Os descritores utilizados foram: Dermatomiosite; Epidemiologia; Miopatias inflamatórias. Na língua inglesa: Dermatomyositis; Epidemiology; Inflammatory myopathies .

\section{RELATO DE CASO}

Paciente D. M S, sexo feminino, 62 anos, do lar procedente de Iúna- ES. Estava em tratamento há 4 meses para depressão com queixas de astenia, prostração e fraqueza, hiporexia, engasgos frequentes, pequena perda ponderal, além de sair da cama somente para necessidades básicas e ter dificuldades até mesmo para pentear os cabelos.

De antecedentes patológico,s ressaltava-se apenas a presença de asma brônquica.

Ao exame físico, apresentava-se em regular estado geral, emagrecida, discreta hipotrofia muscular, fraqueza muscular proximal, mais intensa em membros inferiores, sendo numa escala de 0 a 5, força de grau 3 em membros inferiores e grau $4 \mathrm{em}$ membros superiores. Apresentava ainda lesões elevadas, róseas, simétricas, na face dorsal das articulações interfalangeanas e metacarpofalangeanas. Ainda nas mãos foram encontradas linhas horizontais escurecidas e de aparência suja com fissuras na face lateral dos dedos.

Exames laboratoriais solicitados demonstraram CK total de 3.072 U/L, FAN 1:640 citoplasmático pontilhado fino, VHS $90 \mathrm{~mm} / \mathrm{h}$, Anti JO-1 negativo, Anti MI-2 negativo.

Eletroneuromiografia evidenciou potenciais polifásicos de baixa amplitude e curta duração em diferentes miótomos avaliados nos membros superiores.

Tomografia Computadorizada de Alta Resolução de Tórax apresentou padrão sugestivo de Pneumonia Intersticial Usual em regiões bi-basais e bronquiectasias de tração. Propedêutica inicial para neoplasias foi realizada, porém sem achados.

Iniciado tratamento com Prednisona 60mg dia, e em reavaliação após 15 dias pôde ser observada melhora significativa da fraqueza muscular, sendo a força em membros superiores grau 5 e membros inferiores grau 4, com melhora do estado geral, pequeno ganho ponderal, cessação dos engasgos, e retorno da paciente às atividades habituais da vida diária. A melhora clínica foi acompanhada de redução dos níveis de CK total para $1.500 \mathrm{U} / \mathrm{L}$. Na quarta semana de tratamento com prednisona, paciente 
apresentava melhora completa da força nos quatro membros, melhora também das lesões de pele iniciais, além de apresentar CK total de 983 U/L. Iniciou-se na sexta semana de tratamento a redução gradual da prednisona.

\section{DISCUSSÃO DOS RESULTADOS}

A dermatomiosite além de apresentar início lento e insidioso, não é caracterizada por um episódio clínico identificável, fator que posterga o diagnóstico e permite ampliar as possibilidades de diagnósticos diferenciais. No caso da paciente, as manifestações clínicas se iniciaram há 4 meses até a confirmação do diagnóstico definitivo (IMBODEN et al., 2011).

Alguns sinais clínicos devem ser observados como alarmantes, tais como miopatias inflamatórias, principalmente a fraqueza muscular proximal, associadas a lesões cutâneas, culminando em alterações respiratórias e disfagia, sendo a pneumonia intersticial, a manifestação pulmonar predominante. Já as manifestações cardíacas, como arritmias, insuficiência cardíaca, derrame, podem ser indicativas de mau prognóstico (IMBODEN et al., 2011).

A fraqueza muscular geralmente se inicia nos membros inferiores, podendo acometer também a musculatura de braços, mais comumente a região pélvica e escapular, limitando as simples atividades de vida diária, como subir escadas, levantar da cama e pentear os cabelos. O exame físico utilizado para avaliar a força muscular, em uma escala de 0 a 5 , é o teste manual, encontrando força de grau 3 em membros inferiores e 4 em membros superiores no caso relatado, resultando nas limitações para realizar as tarefas diárias (ORTIGOSA et al., 2008).

As lesões cutâneas podem variar conforme o paciente e suas características podem ser alteradas com o tempo. Porém, lesões patognomônicas de DM, as quais foram encontradas na paciente, são as pápulas de Gottron, caracterizadas por lesões elevadas, simétricas e róseas nas faces dorsal e lateral das articulações interfalangianas e metacarpofalangianas. Outro achado cutâneo, foram linhas horizontais escurecidas, de aspecto sujo com fissuras na face lateral dos dedos, denominadas "mãos de mecânico", bem como máculas violáceas e edemaciadas na região periórbita denominadas 
heliotropo podem ser encontrados; porém, não se confirmaram no caso em questão (GIÁCOMO et al., 2010)

FIGURA 1 - Pápulas de Gottron no dorso das mãos e dedos

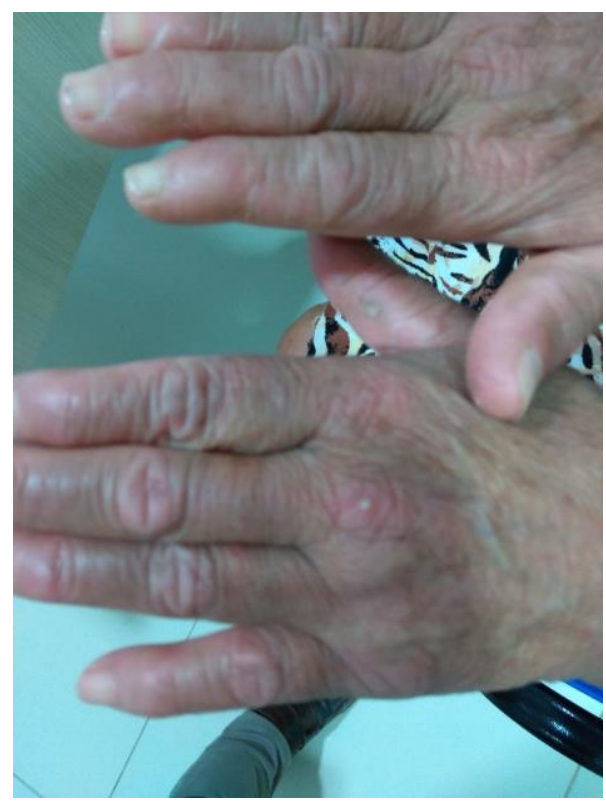

Fonte: Dados da pesquisa.

FIGURA 2 - Mãos de mecânico entre as faces lateral e palmar

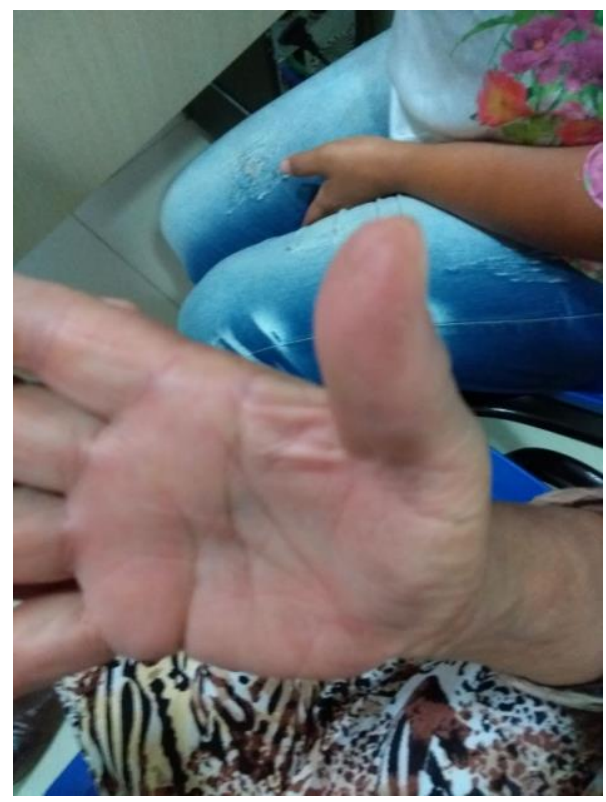

Fonte: Dados da pesquisa.

De acordo Neto et. al. (2010), a dermatomiosite pode ser diagnosticada como definitiva quando apresentar três dos critérios de Bohan e Peter (A, B, C ou D) + 
critério E, os quais se seguem na tabela 1. No caso da paciente apresentada, encontrouse os critérios A, B, C + critério E, definindo o diagnóstico de DM.

QUADRO 1 - Critérios diagnósticos de Boham e Peter

\begin{tabular}{|l|l|}
\hline A & $\begin{array}{l}\text { Fraqueza muscular proximal e simétrica dos músculos das cinturas pélvica e } \\
\text { escapular, flexores anteriores do pescoço, progredindo por semanas a meses, } \\
\text { com ou sem disfagia ou envolvimento de músculos respiratórios. }\end{array}$ \\
\hline B & $\begin{array}{l}\text { Elevação dos níveis séricos de enzimas musculares esqueléticas: } \\
\text { creatinofosfoquinase, aspartato aminotransferase, desidrogenase láctica e } \\
\text { aldolase. }\end{array}$ \\
\hline C & $\begin{array}{l}\text { Eletromiografia característica de miopatia (unidades motoras curtas e pequenas, } \\
\text { fibrilações, ondas pontiagudas positivas, irritabilidade insercional, descargas } \\
\text { repetitivas de alta frequência). }\end{array}$ \\
\hline D & $\begin{array}{l}\text { Biópsia muscular evidenciando necrose, fagocitose, regeneração, atrofia } \\
\text { perifascicular, exsudato inflamatório perivascular }\end{array}$ \\
\hline E & $\begin{array}{l}\text { Alterações cutâneas típicas: • heliotropo com edema periorbitário e eritema } \\
\text { violáceo; } \text { sinal de Gottron: vasculite em articulações de cotovelos, } \\
\text { metacarpofalangeanas e interfalangeanas proximais. }\end{array}$ \\
\hline
\end{tabular}

Fonte: ORTIGOSA et al., 2008.

Os exames laboratoriais podem contribuir para o diagnóstico de dermatomiosite, sendo que o indicador mais sensível, o qual representa miopatia inflamatória, são as alterações nos níveis de creatinocinase (CK), em alguma fase da evolução da doença (GIÁCOMO et al., 2010).

Há ainda o fator antinuclear (FAN), indicativo de doença auto-imune, que pode estar presente no soro de aproximadamente metade dos pacientes com doença muscular inflamatória e foram confirmados na paciente. A presença de auto-anticorpos específicos para miosite servem como indicadores de prognóstico em relação a resposta ao uso de corticoides, sendo o anti-sintetase (anti-Jo-1), o mais comum, porém de resposta moderada ao tratamento; já os anticorpos anti-Mi-2, são praticamente exclusivos de pacientes com DM e são bons preditores de resposta ao tratamento, sendo que ambos os auto anticorpos foram negativos na paciente (BRASIL, 2016). 
Em relação aos exames de imagens, a realização de tomografia computadorizada de tórax foi importante para determinar a presença de pneumonia intersticial bibasais e bronquiectasia. Também deve-se considerar a importância da ressonância magnética para detectar acometimento muscular e de tecidos moles (ORTIGOSA et al., 2008).

A eletroneuromiografia (ENMG) é um exame importante de ser realizado, uma vez que permite diferenciar processos miopátias de neuropatias, bem como determinar a extensão do músculo esquelético acometido. No caso apresentado, a ENMG foi condizente com DM, ao evidenciar potenciais polifásicos de baixa amplitude e curta duração. Outros achados da eletroneuromiografia podem ser ondas positivas pontiagudas, fibrilações musculares, descargas repetitivas e de alta frequência (GIÁCOMO et al., 2010).

A realização da biopsia muscular é importante para determinar o tipo especifico de miopatia inflamatória, sendo que, no caso da DM, a histopatologia revela infiltrado perivascular, contendo linfócitos B e linfócitos T auxiliares CD4+, caracterizando uma microangiopatia. Contudo, em alguns casos, a realização da biopsia pode se apresentar como normal. No caso relatado, não houve a necessidade de realizar biópsia, pois os outros exames realizados possibilitaram diagnosticar a doença (ORTIGOSA et al., 2008).

A conduta adotada após a confirmação do diagnóstico foi o início do tratamento com Prednisona $60 \mathrm{mg} / \mathrm{dia}$ durante 15 dias. Após esse período, foi realizada nova avaliação com a redução dos níveis de CK e melhora da força muscular. Após a sexta semana de tratamento, iniciou-se a redução gradual da Prednisona.

\section{CONCLUSÃO}

No caso relatado juntamente com a análise de publicações, fica evidente a importância da realização do diagnóstico clínico o mais precoce possível, para logo, em seguida, iniciar a terapia com corticoide.

O cuidado na assistência e acompanhamento após a intervenção medicamentosa é essencial para assegurar alívio sintomático e melhoria da qualidade de vida, bem como o monitoramento laboratorial, através dos níveis de CK. 
A importância do controle adequado da doença é importante para reduzir os riscos de complicações, uma vez que a doença pode evoluir para a perda dos reflexos tendinosos e atrofia muscular. Paciente com manifestações pulmonares, podem evoluir para insuficiência respiratória, além de desenvolver cardiomiopatias.

Vale ressaltar que a dematomiosite por ser uma doença auto-imune, não apresenta cura definitiva; porém, quando tratada adequadamente, o que inclui uso correto dos glicocorticóides, para evitar toxicidade e efeitos colaterais, pode ser controlada com êxito.

Sendo assim, quanto mais precoce e assertivo for o diagnóstico, bem como a implementação do tratamento adequado, melhor será o prognóstico e menores serão as chances de evolução grave da doença (NETO et al., 2010).

\section{Referências}

BRASIL. Ministério da Saúde. Protocolo Clínico e Diretrizes Terapêuticas Dermatomiosite e Polimiosite. Relatório de Recomendação. Brasília: Ministério da Saúde, 2016.

GIACOMO, C. G. et. al. Atualização em dermatomiosite. Revista Sociedade Brasileira Clínica Médica. São Paulo. v. 8, n.5, PP 434-439, set./out. 2010.

IMBODEN. J. B. et. al. Current Reumatologia: Diagnóstico e tratamento. Porto Alegre: AMGH. 2. ed., 2011.

NETO, N. S. R. et. al. Dermatomiosite juvenil: revisão e atualização em patogênese e tratamento. Revista Brasileira de Reumatologia, São Paulo. v.50, n.3, pp. 299-312, Jun 2010. Disponível em: < http://www.scielo.br/scielo.php?script=sci_arttext\&pid=S048250042010000300010\&lng=en\&nrm=iso > Acesso em: 19 de Jan. 2018.

ORTIGOSA, L. C. M. et. al. Dermatomiosite. Revista de Associação Brasileira de Dermatologia. Rio de Janeiro. v. 83, n. 3, pp. 247-259, Jun 2008.

PEREIRA, F. C. et al. Dermatopolimiosite juvenil: relato de caso. Rev. Med. Res. 2011; v. 13, n.2 122-126.

VILAS, A. P. et. al. Um caso de dermatomiosite numa mulher jovem. Revista Medicina Interna. v. 6, n.3, pp. 164-167, 2009. 